\title{
Movability and Uniform Movability of Shape Morphisms by
}

\author{
P. S. GEVORGYAN and I. POP \\ Presented by Czesław BESSAGA
}

\begin{abstract}
Summary. The purpose of this paper is to define some notions of movability for morphisms of inverse systems which extend the movability properties of inverse systems and which are compatible with the equivalence relations which define pro-morphisms and shape morphisms. Some properties and applications are given.
\end{abstract}

1. Introduction. The notion of movability for metric compacta was introduced by K. Borsuk [2] as an important shape invariant. The movable spaces are a generalization of spaces having the shape of ANR's. The movability assumption allows a series of important results in algebraic topology (like the Whitehead and Hurewicz theorems) to remain valid when the homotopy pro-groups are replaced by the corresponding shape groups. The term "movability" comes from the geometric interpretation of the definition in the compact case: if $X$ is a compactum lying in a space $M \in \mathrm{AR}$, one says that $X$ is movable if for every neighborhood $U$ of $X$ in $M$ there exists a neighborhood $V \subset U$ of $X$ such that for every neighborhood $W \subset U$ of $X$ there is a homotopy $H: V \times[0,1] \rightarrow U$ such that $H(x, 0)=x$ and $H(x, 1) \in W$ for every $x \in V$. One shows that the choice of $M \in \mathrm{AR}$ is irrelevant [2]. After the notion of movability had been expressed in terms of ANR-systems [11, [12, it became clear that one could define it in arbitrary pro-categories. The definition of a movable object in an arbitrary pro-category and that of uniform movability were both given by Maria Moszyńska [14]. In the book

2010 Mathematics Subject Classification: Primary 55P55; Secondary 54C56.

Key words and phrases: movability and co-movability (uniform) of morphisms of inverse systems, of pro-morphisms, of shape morphisms and maps.

Received 25 February 2016; revised 19 June 2016.

Published online 22 August 2016. 
of Sibe Mardešić and Jack Segal [12] all these approaches and applications of various types of movability are discussed.

Some categorical approaches to movability in shape theory were given by P. S. Gevorgyan [7], 8], I. Pop [15], P. S. Gevorgyan \& I. Pop [9], and T. A. Avakyan \& P. S. Gevorgyan [1].

The approach of this paper started from the idea that some results in shape theory, valid under some conditions of movability for inverse systems or shape objects, could be obtained under weak conditions. More precisely, the idea was to define some movability notions for morphisms of inverse systems, so as to recover, when these morphisms are identity morphisms, the definitions of the usual movability properties for pro-objects and shape objects. Besides the classical case of movability of pro-objects and shape objects, some notions of movability appear in the papers of T. Yagasaki [19, [20] about shape fibrations, of Z. Cerin [3] on Lefschetz movable maps, and of D. A. Edwards and P. Tulley MacAuley [6] about a shape theory of a map.

Unfortunately, these approaches are just particular cases and they do not deal with the movability of shape morphisms in the general case of an abstract shape theory. The second author of this paper already approached this problem in two previous articles [16, [17] in which some properties of movability for morphisms of inverse systems and for pro-morphisms were defined and studied. However, those properties could not be extended to shape morphisms and maps.

In the present paper this problem is solved by a slight but useful modification of the previous notions of movability and uniform movability for morphisms of inverse systems, the new definitions being compatible with the relations which define pro-morphisms and shape morphisms. In this way we obtain satisfactory notions of movability (simple and uniform) for shape morphisms and in particular for maps. "Dual" notions of co-movability (simple and uniform) are defined. Some properties and applications are given.

\section{Movable morphisms of inverse systems and movable shape} morphisms. All sets of indices considered are supposed to be cofinite directed sets. This condition is not restrictive (cf. [12, Ch. I, §1.2]).

First we recall from [12, Ch. II, §6.1] the following definition.

Definition 2.1. An object $\mathbf{X}=\left(X_{\lambda}, p_{\lambda \lambda^{\prime}}, \Lambda\right)$ of pro-C is movable provided every $\lambda \in \Lambda$ admits a $\lambda^{\prime} \geq \lambda$ (called a movability index of $\lambda$ ) such that each $\lambda^{\prime \prime} \geq \lambda$ admits a morphism $r: X_{\lambda^{\prime}} \rightarrow X_{\lambda^{\prime \prime}}$ of $\mathcal{C}$ which satisfies

$$
p_{\lambda \lambda^{\prime \prime}} \circ r=p_{\lambda \lambda^{\prime}},
$$

i.e., makes the following diagram commutative: 


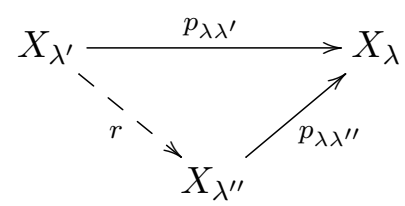

Definition 2.2. Let $\mathbf{X}=\left(X_{\lambda}, p_{\lambda \lambda^{\prime}}, \Lambda\right)$ and $\mathbf{Y}=\left(Y_{\mu}, q_{\mu \mu^{\prime}}, M\right)$ be inverse systems in a category $\mathcal{C}$ and $\left(f_{\mu}, \phi\right): \mathbf{X} \rightarrow \mathbf{Y}$ a morphism of inverse systems. We say that the morphism $\left(f_{\mu}, \phi\right)$ is movable if every $\mu \in M$ admits a $\lambda \in \Lambda$ with $\lambda \geq \phi(\mu)$ such that each $\mu^{\prime} \in M$ with $\mu^{\prime} \geq \mu$ admits a morphism $u: X_{\lambda} \rightarrow Y_{\mu^{\prime}}$ in $\mathcal{C}$ which satisfies

$$
f_{\mu} \circ p_{\phi(\mu) \lambda}=q_{\mu \mu^{\prime}} \circ u,
$$

i.e., makes the following diagram commutative:

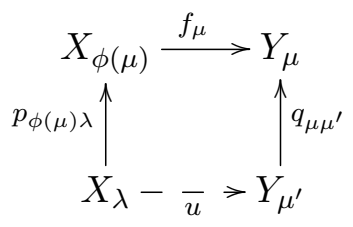

The index $\lambda$ is called a movability index (MI) of $\mu$ with respect to $\left(f_{\mu}, \phi\right)$.

The composition $f_{\mu} \circ p_{\phi(\mu) \lambda}$ for $\lambda \geq \phi(\mu)$ is denoted by $f_{\mu \lambda}$ (cf. [12, Ch. II, $\S 2.1])$. With this notation the relation $(2.2)$ becomes $f_{\mu \lambda}=q_{\mu \mu^{\prime}} \circ u$.

REMARK 2.3. If $\lambda$ is a movability index for $\mu$ with respect to $\left(f_{\mu}, \phi\right)$, then so is any $\tilde{\lambda}>\lambda$.

TheOREM 2.4. An inverse system $\mathbf{X}=\left(X_{\lambda}, p_{\lambda \lambda^{\prime}}, \Lambda\right)$ is movable if and only if the identity morphism $1_{\mathbf{X}}$ is movable.

Proof. Suppose $\mathbf{X}=\left(X_{\lambda}, p_{\lambda \lambda^{\prime}}, \Lambda\right)$ is movable. Consider an arbitrary $\lambda \in \Lambda$. Note that a movability index $\lambda^{\prime} \geq \lambda$ is also a movability index of $\lambda$ with respect to $1_{\mathbf{X}}: \mathbf{X} \rightarrow \mathbf{X}$.

Conversely, suppose $1_{\mathbf{X}}: \mathbf{X} \rightarrow \mathbf{X}$ is a movable morphism. Note that for any $\lambda \in \Lambda$ a movability index $\lambda^{\prime} \geq \lambda$ of $\lambda$ with respect to $1_{\mathbf{X}}$ is also a movability index for the inverse system $\mathbf{X}=\left(X_{\lambda}, p_{\lambda \lambda^{\prime}}, \Lambda\right)$.

Theorem 2.5. Let $\mathbf{X}=\left(X_{\lambda}, p_{\lambda \lambda^{\prime}}, \Lambda\right), \mathbf{Y}=\left(Y_{\mu}, q_{\mu \mu^{\prime}}, M\right)$ and $\mathbf{Z}=$ $\left(Z_{\nu}, r_{\nu \nu^{\prime}}, N\right)$ be inverse systems in the category $\mathcal{C}$ and let $\left(f_{\mu}, \phi\right): \mathbf{X} \rightarrow \mathbf{Y}$ and $\left(g_{\nu}, \psi\right): \mathbf{Y} \rightarrow \mathbf{Z}$ be morphisms of inverse systems. Suppose that $\left(g_{\nu}, \psi\right)$ is movable. Then so is the composition $\left(h_{\nu}, \chi\right)=\left(g_{\nu}, \psi\right) \circ\left(f_{\mu}, \phi\right)$.

Proof. Recall that $\chi=\phi \circ \psi$ and $h_{\nu}=g_{\nu} \circ f_{\psi(\nu)}$. If $\left(g_{\nu}, \psi\right)$ is movable and $\nu \in N$, then for a movability index $\mu \in M$ of $\nu \in N$ with $\mu \geq \psi(\nu)$, an index $\nu^{\prime} \in N$ with $\nu^{\prime} \geq \nu$ admits a morphism $u: Y_{\mu} \rightarrow Z_{\nu^{\prime}}$ in $\mathcal{C}$ such that 
$g_{\nu} \circ q_{\psi(\nu) \mu}=r_{\nu \nu^{\prime}} \circ u:$

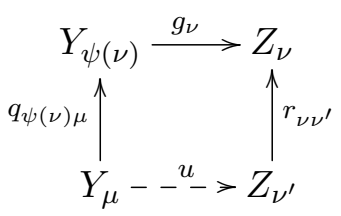

Now consider $\lambda \in \Lambda$ with $\lambda \geq \phi(\mu)$ and $\lambda \geq \phi(\psi(\nu))$ such that $f_{\psi(\nu)} \circ$ $p_{\phi(\psi(\nu)) \lambda}=q_{\psi(\nu) \mu} \circ f_{\mu} \circ p_{\phi(\mu) \lambda}$. Then we can consider the morphism $u^{\prime}:=$ $u \circ f_{\mu} \circ p_{\phi(\mu) \lambda}: X_{\lambda} \rightarrow Z_{\nu^{\prime}}$, for which we obtain

$$
\begin{aligned}
r_{\nu \nu^{\prime}} \circ u^{\prime} & =\left(r_{\nu \nu^{\prime}} \circ u\right) \circ f_{\mu} \circ p_{\phi(\mu) \lambda}=g_{\nu} \circ q_{\psi(\nu)} \circ f_{\mu} \circ p_{\phi(\mu) \lambda} \\
& =g_{\nu} \circ f_{\psi(\nu)} \circ p_{\phi(\psi(\nu))}=h_{\nu} \circ p_{\chi(\nu) \lambda} .
\end{aligned}
$$

COROllary 2.6. Let $\mathbf{X}=\left(X_{\lambda}, p_{\lambda \lambda^{\prime}}, \Lambda\right)$ be an arbitrary inverse system and $\mathbf{Y}=\left(Y_{\mu}, q_{\mu \mu^{\prime}}, M\right)$ be movable. Then any morphism $\left(f_{\mu}, \phi\right): \mathbf{X} \rightarrow \mathbf{Y}$ is movable.

Proof. Since $\left(f_{\mu}, \phi\right)=1_{\mathbf{Y}} \circ\left(f_{\mu}, \phi\right)$ and $1_{\mathbf{Y}}: \mathbf{Y} \rightarrow \mathbf{Y}$ is a movable morphism by Theorem 2.4. $\left(f_{\mu}, \phi\right)$ is also movable according to Theorem 2.5.

Theorem 2.7. Let $\mathbf{X}=\left(X_{\lambda}, p_{\lambda \lambda^{\prime}}, \Lambda\right), \mathbf{Y}=\left(Y_{\mu}, q_{\mu \mu^{\prime}}, M\right)$ and $\mathbf{Z}=$ $\left(Z_{\nu}, r_{\nu \nu^{\prime}}, N\right)$ be inverse systems in the category $\mathcal{C}$, and let $\left(f_{\mu}, \phi\right): \mathbf{X} \rightarrow \mathbf{Y}$ and $\left(g_{\nu}, \psi\right): \mathbf{Y} \rightarrow \mathbf{Z}$ be morphisms of inverse systems. Suppose that $\left(f_{\mu}, \phi\right)$ is movable. Then so is the composition $\left(h_{\nu}, \chi\right)=\left(g_{\nu}, \psi\right) \circ\left(f_{\mu}, \phi\right)$.

Proof. For any $\nu \in N$, consider a movability index $\lambda$ of $\psi(\nu), \lambda \geq$ $\phi(\psi(\nu))$, with respect to $\left(f_{\mu}, \phi\right)$. Let us prove that $\lambda$ is a movability index of $\nu$ with respect to $\left(h_{\nu}, \chi\right)$.

Let $\nu^{\prime} \in N$ with $\nu^{\prime} \geq \nu$. Consider $\mu^{\prime} \geq \psi\left(\nu^{\prime}\right), \psi(\nu)$ such that

$$
r_{\nu \nu^{\prime}} \circ g_{\nu^{\prime}} \circ q_{\psi\left(\nu^{\prime}\right) \mu^{\prime}}=g_{\nu} \circ q_{\psi(\nu) \mu^{\prime}} .
$$

For $q_{\psi(\nu) \mu^{\prime}}$ by the movability of $\left(f_{\mu}, \phi\right): \mathbf{X} \rightarrow \mathbf{Y}$ there exists a morphism $u: X_{\lambda} \rightarrow Y_{\mu^{\prime}}$ such that

$$
f_{\psi(\nu)} \circ p_{\phi(\psi(\nu)) \lambda}=q_{\psi(\nu) \mu^{\prime}} \circ u .
$$

Define $U: X_{\lambda} \rightarrow Z_{\nu^{\prime}}$ by

$$
U=g_{\nu^{\prime}} \circ q_{\psi\left(\nu^{\prime}\right) \mu^{\prime}} \circ u .
$$

Now we have

$$
\begin{aligned}
r_{\nu \nu^{\prime}} \circ U & =r_{\nu \nu^{\prime}} \circ g_{\nu^{\prime}} \circ q_{\psi\left(\nu^{\prime}\right) \mu^{\prime}} \circ u=g_{\nu} \circ q_{\psi(\nu) \mu^{\prime}} \circ u \\
& =g_{\nu} \circ q_{\psi(\nu) \mu} \circ f_{\mu} \circ p_{\phi(\mu) \lambda}=g_{\nu} \circ f_{\psi(\nu)} \circ p_{\chi(\nu) \lambda} \\
& =h_{\nu} \circ p_{\chi(\nu) \lambda} .
\end{aligned}
$$


Corollary 2.8. Let $\mathbf{X}=\left(X_{\lambda}, p_{\lambda \lambda^{\prime}}, \Lambda\right)$ be movable and $\mathbf{Y}=\left(Y_{\mu}, q_{\mu \mu^{\prime}}, M\right)$ be an arbitrary inverse system. Then any morphism $\left(f_{\mu}, \phi\right): \mathbf{X} \rightarrow \mathbf{Y}$ is movable.

Proof. Since $\left(f_{\mu}, \phi\right)=\left(f_{\mu}, \phi\right) \circ 1_{\mathbf{X}}$ and $1_{\mathbf{X}}: \mathbf{X} \rightarrow \mathbf{X}$ is a movable morphism by Theorem 2.4. $\left(f_{\mu}, \phi\right)$ is also movable according to Theorem 2.7 .

Proposition 2.9. Let $\left(f_{\mu}, \phi\right),\left(f_{\mu}^{\prime}, \phi^{\prime}\right): \mathbf{X}=\left(X_{\lambda}, p_{\lambda \lambda^{\prime}}, \Lambda\right) \rightarrow \mathbf{Y}=$ $\left(Y_{\mu}, q_{\mu \mu^{\prime}}, M\right)$ be equivalent morphisms of inverse systems. If $\left(f_{\mu}, \phi\right)$ is movable then so is $\left(f_{\mu}^{\prime}, \phi^{\prime}\right)$.

Proof. Let $\mu \in M$ with $\lambda$ a movability index for $\mu$ with respect to $\left(f_{\mu}, \phi\right)$. Now by the definition of the equivalence relation between morphisms of inverse systems (see [12, Ch. I, §1.1]), there exists $\lambda^{\prime} \geq \phi(\mu), \phi^{\prime}(\mu)$ such that

$$
f_{\mu} \circ p_{\phi(\mu) \lambda^{\prime}}=f_{\mu}^{\prime} \circ p_{\phi^{\prime}(\mu) \lambda^{\prime}}
$$

and we can suppose that $\lambda^{\prime} \geq \lambda$, so that we refer to $\lambda^{\prime}$ as a movability index of $\mu$ with respect to $\left(f_{\mu}, \phi\right)$. Then $\lambda^{\prime}$ is also a movability index for $\mu$ with respect to $\left(f_{\mu}^{\prime}, \phi^{\prime}\right)$. Let $\mu^{\prime} \in M$ with $\mu^{\prime} \geq \mu$. Then there exists a morphism $u: X_{\lambda^{\prime}} \rightarrow Y_{\mu^{\prime}}$ such that $f_{\mu \lambda^{\prime}}=q_{\mu \mu^{\prime}} \circ u$.

But by 2.3 we have $f_{\mu \lambda^{\prime}}=f_{\mu \lambda^{\prime}}^{\prime}$, so that $f_{\mu \lambda^{\prime}}^{\prime}=q_{\mu \mu^{\prime}} \circ u$.

Recall that the pro-category pro- $\mathcal{C}$ of a category $\mathcal{C}$ has as objects all inverse systems $\mathbf{X}$ in $\mathcal{C}$ (over all directed sets $\Lambda$ ), and as morphisms $\mathbf{f}: \mathbf{X} \rightarrow \mathbf{Y}$ the equivalence classes of morphisms $\left(f_{\mu}, \phi\right): \mathbf{X} \rightarrow \mathbf{Y}$ of inverse systems with respect to the equivalence relation $\sim$ considered above.

Thanks to Proposition 2.9 we can make the following definition.

Definition 2.10. A morphism $\mathbf{f}: \mathbf{X} \rightarrow \mathbf{Y}$ in pro- $\mathcal{C}$ is called movable if $\mathbf{f}$ admits a representation $\left(f_{\mu}, \phi\right): \mathbf{X} \rightarrow \mathbf{Y}$ which is movable.

The next theorem follows from Theorems 2.5 and 2.7 and Corollaries 2.6 and 2.8 .

THEOREM 2.11. A (pre-or post-) composition of an arbitrary pro-morphism with a movable pro-morphism is a movable pro-morphism. In particular, if for a pro-morphism $\mathbf{f}: \mathbf{X} \rightarrow \mathbf{Y}$, one of $\mathbf{X}$ or $\mathbf{Y}$ is a movable system, then $\mathbf{f}$ is a movable pro-morphism.

Corollary 2.12. Let $\mathbf{X}=\left(X_{\lambda}, p_{\lambda \lambda^{\prime}}, \Lambda\right)$ and $\mathbf{Y}=\left(Y_{\mu}, q_{\mu \mu^{\prime}}, M\right)$ in pro-C. If $\mathbf{Y}$ is movable and $\mathbf{X}$ is dominated by $\mathbf{Y}$ in pro-C , then $\mathbf{X}$ is also movable.

Proof. By assumption there exist pro-morphisms $\mathbf{f}: \mathbf{X} \rightarrow \mathbf{Y}$ and $\mathbf{g}:$ $\mathbf{Y} \rightarrow \mathbf{X}$ such that $\mathbf{g} \circ \mathbf{f}=1_{\mathbf{X}}$. Because $\mathbf{Y}$ is movable, by Theorem 2.11 it follows that $\mathbf{f}, \mathbf{g}$ and $1_{\mathbf{X}}$ are movable pro-morphisms, and hence $\mathbf{X}$ is movable by Theorem 2.4 . 
REMARK 2.13. Corollary 2.12 is a new proof of an important result in the theory of shape (see [12, Theorem 1, Ch. II, §6.1]).

Based on Proposition 2.9, we can introduce the following definition.

Definition 2.14. A shape morphism $F: X \rightarrow Y$ is movable if it can be represented by a movable pro-morphism $\mathbf{f}: \mathbf{X} \rightarrow \mathbf{Y}$.

\section{Uniformly movable shape morphisms}

Definition 3.1. Let $\mathbf{X}=\left(X_{\lambda}, p_{\lambda \lambda^{\prime}}, \Lambda\right)$ and $\mathbf{Y}=\left(Y_{\mu}, q_{\mu \mu^{\prime}}, M\right)$ be inverse systems in a category $\mathcal{C}$, and $\left(f_{\mu}, \phi\right): \mathbf{X} \rightarrow \mathbf{Y}$ a morphism of inverse systems. We say that $\left(f_{\mu}, \phi\right)$ is uniformly movable if every $\mu \in M$ admits $\lambda \in \Lambda$ with $\lambda \geq \phi(\mu)$ (called a uniform movability index of $\mu$ with respect to $\left(f_{\mu}, \phi\right)$ ) such that there is a morphism of inverse systems $\mathbf{u}: X_{\lambda} \rightarrow \mathbf{Y}$ satisfying

$$
f_{\mu \lambda}=\mathbf{q}_{\mu} \circ \mathbf{u}
$$

i.e., the following diagram commutes:

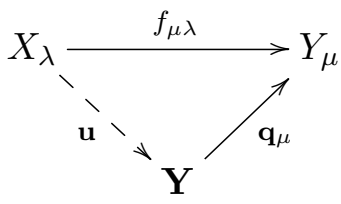

where $\mathbf{q}_{\mu}: \mathbf{Y} \rightarrow Y_{\mu}$ is the morphism of inverse systems given by $1_{\mu}$ : $Y_{\mu} \rightarrow Y_{\mu}$.

REMARK 3.2. If $\lambda$ is a uniform movability index, then so is any $\tilde{\lambda}>\lambda$.

REMARK 3.3. Note that the morphism $\mathbf{u}: X_{\lambda} \rightarrow \mathbf{Y}$ determines for every $\mu_{1} \in M$ a morphism $u_{\mu_{1}}: X_{\lambda} \rightarrow Y_{\mu_{1}}$ in $\mathcal{C}$ such that for $\mu_{1} \leq \mu_{2}$ we have $q_{\mu_{1} \mu_{2}} \circ u_{\mu_{2}}=u_{\mu_{1}}$ and $u_{\mu}=f_{\mu \lambda}$. In particular, for $\mu^{\prime} \in M$ with $\mu^{\prime} \geq \mu$, we have $q_{\mu \mu^{\prime}} \circ u_{\mu^{\prime}}=u_{\mu}=f_{\mu \lambda}$, so that uniform movability of morphisms implies movability.

TheORem 3.4. An inverse system $\mathbf{X}=\left(X_{\lambda}, p_{\lambda \lambda^{\prime}}, \Lambda\right)$ is uniformly movable if and only if the identity morphism $1_{\mathbf{X}}$ is uniformly movable.

Proof. Suppose $\mathbf{X}=\left(X_{\lambda}, p_{\lambda \lambda^{\prime}}, \Lambda\right)$ is uniformly movable. Let $\lambda \in \Lambda$. Note that a uniform movability index $\lambda^{\prime} \geq \lambda$ is also a uniform movability index of $\lambda$ with respect to $1_{\mathbf{X}}: \mathbf{X} \rightarrow \mathbf{X}$.

Conversely, suppose $1 \mathbf{X}: \mathbf{X} \rightarrow \mathbf{X}$ is a uniformly movable morphism. Note that for any $\lambda \in \Lambda$ a uniform movability index $\lambda^{\prime} \geq \lambda$ of $\lambda$ with respect to $1_{\mathbf{X}}$ is also a uniform movability index for $\mathbf{X}=\left(X_{\lambda}, p_{\lambda \lambda^{\prime}}, \Lambda\right)$.

Theorem 3.5. Let $\mathbf{X}=\left(X_{\lambda}, p_{\lambda \lambda^{\prime}}, \Lambda\right), \mathbf{Y}=\left(Y_{\mu}, q_{\mu \mu^{\prime}}, M\right), \mathbf{Z}=\left(Z_{\nu}, r_{\nu \nu^{\prime}}, N\right)$ be inverse systems in the category $\mathcal{C}$, and let $\left(f_{\mu}, \phi\right): \mathbf{X} \rightarrow \mathbf{Y}$ and $\left(g_{\nu}, \psi\right)$ : 
$\mathbf{Y} \rightarrow \mathbf{Z}$ be morphisms of inverse systems. If $\left(g_{\nu}, \psi\right)$ is uniformly movable, then so is $\left(h_{\nu}, \chi\right)=\left(g_{\nu}, \psi\right) \circ\left(f_{\mu}, \phi\right)$.

Proof. We use the notation from the proof of Theorem 2.5 replacing $r_{\nu \nu^{\prime}}: Z_{\nu^{\prime}} \rightarrow Z_{\nu}$ by $\mathbf{r}_{\nu}: \mathbf{Z} \rightarrow Z_{\nu}$ and $u: Y_{\mu} \rightarrow Z_{\nu^{\prime}}$ by $\mathbf{u}: Y_{\mu} \rightarrow \mathbf{Z}$. Then we have $g_{\nu} \circ q_{\psi(\nu) \mu}=\mathbf{r}_{\nu} \circ \mathbf{u}$. And by defining $\mathbf{u}^{\prime}=\mathbf{u} \circ f_{\mu \lambda}: X_{\lambda} \rightarrow \mathbf{Z}$, we obtain $\mathbf{r}_{\nu} \circ \mathbf{u}^{\prime}=h_{\nu \lambda}$.

Corollary 3.6. Let $\mathbf{X}=\left(X_{\lambda}, p_{\lambda \lambda^{\prime}}, \Lambda\right)$ be an arbitrary inverse system and $\mathbf{Y}=\left(Y_{\mu}, q_{\mu \mu^{\prime}}, M\right)$ be uniformly movable. Then any morphism $\left(f_{\mu}, \phi\right)$ : $\mathbf{X} \rightarrow \mathbf{Y}$ is uniformly movable.

Proof. Since $\left(f_{\mu}, \phi\right)=1_{\mathbf{Y}} \circ\left(f_{\mu}, \phi\right)$ and $1_{Y}: Y \rightarrow Y$ is uniformly movable by Theorem 3.4, we conclude that $\left(f_{\mu}, \phi\right)$ is also uniformly movable according to Theorem 3.5 .

Theorem 3.7. Let $\mathbf{X}=\left(X_{\lambda}, p_{\lambda \lambda^{\prime}}, \Lambda\right), \mathbf{Y}=\left(Y_{\mu}, q_{\mu \mu^{\prime}}, M\right)$ and $\mathbf{Z}=$ $\left(Z_{\nu}, r_{\nu \nu^{\prime}}, N\right)$ be inverse systems in the category $\mathcal{C}$, and let $\left(f_{\mu}, \phi\right): \mathbf{X} \rightarrow \mathbf{Y}$ and $\left(g_{\nu}, \psi\right): \mathbf{Y} \rightarrow \mathbf{Z}$ be morphisms of inverse systems. Suppose that $\left(f_{\mu}, \phi\right)$ is uniformly movable. Then so is $\left(h_{\nu}, \chi\right)=\left(g_{\nu}, \psi\right) \circ\left(f_{\mu}, \phi\right)$.

Proof. In the notation from the proof of Theorem 2.7, there exists $\mathbf{u}$ : $X_{\lambda} \rightarrow \mathbf{Y}$ such that $f_{\psi(\nu) \lambda}=\mathbf{q}_{\psi(\nu)} \circ \mathbf{u}$. Then for $\mathbf{U}=\mathbf{g} \circ \mathbf{u}: X_{\lambda} \rightarrow \mathbf{Z}$, we have $h_{\nu \lambda}=g_{\nu} \circ f_{\psi(\nu) \lambda}=\mathbf{r}_{\nu} \circ \mathbf{U}$.

Corollary 3.8. Let $\mathbf{X}=\left(X_{\lambda}, p_{\lambda \lambda^{\prime}}, \Lambda\right)$ be uniformly movable and $\mathbf{Y}=$ $\left(Y_{\mu}, q_{\mu \mu^{\prime}}, M\right)$ be an arbitrary inverse system. Then any morphism $\left(f_{\mu}, \phi\right)$ : $\mathbf{X} \rightarrow \mathbf{Y}$ is uniformly movable.

Proof. Since $\left(f_{\mu}, \phi\right)=\left(f_{\mu}, \phi\right) \circ 1_{\mathbf{X}}$ and $1_{\mathbf{X}}: \mathbf{X} \rightarrow \mathbf{X}$ is uniformly movable by Theorem 3.4. $\left(f_{\mu}, \phi\right)$ is also uniformly movable according to Theorem 3.7

Proposition 3.9. Let $\left(f_{\mu}, \phi\right),\left(f_{\mu}^{\prime}, \phi^{\prime}\right): \mathbf{X}=\left(X_{\lambda}, p_{\lambda \lambda^{\prime}}, \Lambda\right) \rightarrow \mathbf{Y}=$ $\left(Y_{\mu}, q_{\mu \mu^{\prime}}, M\right)$ be two equivalent morphisms of inverse systems. If $\left(f_{\mu}, \phi\right)$ is uniformly movable then so is $\left(f_{\mu}^{\prime}, \phi^{\prime}\right)$.

Proof. We use the notation from the proof of Proposition 2.9. For $\mu \in M$ let $\lambda^{\prime} \in \Lambda$ be a uniform movability index with respect to $\left(f_{\mu}, \phi\right)$ such that (2.3) holds. This means $f_{\mu \lambda^{\prime}}=f_{\mu \lambda^{\prime}}^{\prime}$ and therefore $f_{\mu \lambda^{\prime}}^{\prime}=f_{\mu \lambda^{\prime}}=\mathbf{q}_{\mu} \circ \mathbf{u}$.

Thanks to Proposition 3.9 we can make the following definition.

Definition 3.10. A morphism $\mathbf{f}: \mathbf{X} \rightarrow \mathbf{Y}$ in a pro-category pro- $\mathcal{C}$ is called uniformly movable if $\mathbf{f}$ admits a representation $\left(f_{\mu}, \phi\right): \mathbf{X} \rightarrow \mathbf{Y}$ which is uniformly movable.

The next theorem follows from Theorems 3.5 and 3.7 and Corollaries 3.6 and 3.8 . 
THEOREM 3.11. A (pre- or post-) composition of an arbitrary pro-morphism with a uniformly movable pro-morphism is a uniformly movable promorphism. In particular, if for a pro-morphism $\mathbf{f}: \mathbf{X} \rightarrow \mathbf{Y}$, one of $\mathbf{X}$ or $\mathbf{Y}$ is a uniformly movable system, then $\mathbf{f}$ is a uniformly movable pro-morphism.

Corollary 3.12. Let $\mathbf{X}=\left(X_{\lambda}, p_{\lambda \lambda^{\prime}}, \Lambda\right)$ and $\mathbf{Y}=\left(Y_{\mu}, q_{\mu \mu^{\prime}}, M\right)$ in pro-C. If $\mathbf{Y}$ is uniformly movable and $\mathbf{X}$ is dominated by $\mathbf{Y}$ in pro-C , then $\mathbf{X}$ is uniformly movable.

Proof. By assumption there exist pro-morphisms $\mathbf{f}: \mathbf{X} \rightarrow \mathbf{Y}$ and $\mathbf{g}$ : $\mathbf{Y} \rightarrow \mathbf{X}$ such that $\mathbf{g} \circ \mathbf{f}=1_{\mathbf{X}}$. Because $\mathbf{Y}$ is uniformly movable, Theorem 3.11 shows that $\mathbf{f}, \mathbf{g}$ and $1_{\mathbf{X}}$ are uniformly movable pro-morphisms, and hence $\mathbf{X}$ is uniformly movable by Theorem 3.4 .

REMARK 3.13. Corollary 3.12 is a new proof of an important result in the theory of shape (see [12, Theorem 2, Ch. II, §6.1]).

Definition 3.14. A shape morphism $F: X \rightarrow Y$ is uniformly movable if it can be represented by a uniformly movable pro-morphism $\mathbf{f}: \mathbf{X} \rightarrow \mathbf{Y}$.

\section{Co-movability}

Definition 4.1. Let $\mathbf{X}=\left(X_{\lambda}, p_{\lambda \lambda^{\prime}}, \Lambda\right)$ and $\mathbf{Y}=\left(Y_{\mu}, q_{\mu \mu^{\prime}}, M\right)$ be inverse systems in a category $\mathcal{C}$, and $\left(f_{\mu}, \phi\right): \mathbf{X} \rightarrow \mathbf{Y}$ a morphism of inverse systems. We say that $\left(f_{\mu}, \phi\right)$ is co-movable provided every $\mu \in M$ admits $\lambda \in \Lambda$ with $\lambda \geq \phi(\mu)$ (called a co-movability index of $\mu$ relative to $\left(f_{\mu}, \phi\right)$ ) such that each $\lambda^{\prime} \geq \phi(\mu)$ admits a morphism $r: X_{\lambda} \rightarrow X_{\lambda^{\prime}}$ of $\mathcal{C}$ which satisfies

$$
f_{\mu \lambda}=f_{\mu \lambda^{\prime}} \circ r
$$

i.e., makes the outside diagram below commutative:

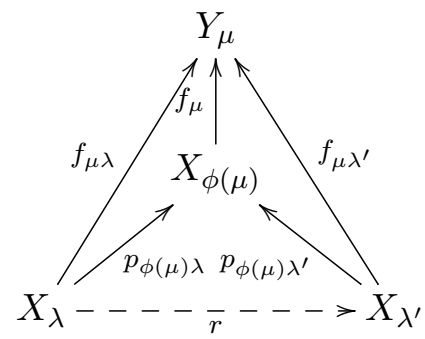

Definition 4.2. Let $\mathbf{X}=\left(X_{\lambda}, p_{\lambda \lambda^{\prime}}, \Lambda\right)$ and $\mathbf{Y}=\left(Y_{\mu}, q_{\mu \mu^{\prime}}, M\right)$ be inverse systems in a category $\mathcal{C}$, and $\left(f_{\mu}, \phi\right): \mathbf{X} \rightarrow \mathbf{Y}$ a morphism of inverse systems. We say that $\left(f_{\mu}, \phi\right)$ is uniformly co-movable provided every $\mu \in M$ admits $\lambda \in \Lambda$ with $\lambda \geq \phi(\mu)$ (called a uniform co-movability index of $\mu$ relative to $\left.\left(f_{\mu}, \phi\right)\right)$ such that there is a morphism $\mathbf{r}: X_{\lambda} \rightarrow \mathbf{X}$ in pro- $\mathcal{C}$ satisfying

$$
f_{\mu \lambda}=\mathbf{f}_{\mu} \circ \mathbf{r}
$$


i.e., making the outside diagram below commutative:

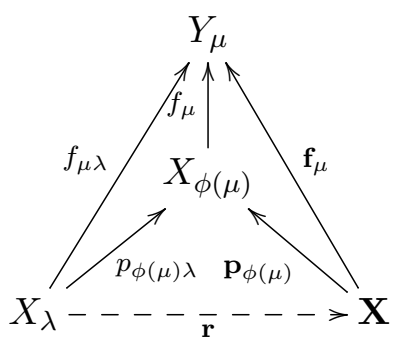

where $\mathbf{f}_{\mu}=f_{\mu} \circ \mathbf{p}_{\phi(\mu)}$.

REMARK 4.3. The pro-morphism $\mathbf{r}: X_{\lambda} \rightarrow \mathbf{X}$ is given by some morphisms $r_{\lambda^{\prime}}: X_{\lambda} \rightarrow X_{\lambda^{\prime}}$ such that if $\lambda_{1}^{\prime} \leq \lambda_{2}^{\prime}$ then $r_{\lambda_{1}^{\prime}}=p_{\lambda_{1}^{\prime} \lambda_{2}^{\prime}} \circ r_{\lambda_{2}^{\prime}}$. The relation $f_{\mu \lambda}=\mathbf{f}_{\mu} \circ \mathbf{r}$ means $f_{\mu \lambda}=f_{\mu} \circ r_{\phi(\mu)}$. Therefore, $\lambda^{\prime} \geq \phi(\mu)$ implies $f_{\mu \lambda}=f_{\mu} \circ p_{\phi(\mu) \lambda^{\prime}} \circ r_{\lambda^{\prime}}=f_{\mu \lambda^{\prime}} \circ r_{\lambda^{\prime}}$. Thus uniform co-movability implies co-movability.

Remark 4.4. Suppose $\left(f_{\mu}, \phi\right): \mathbf{X}=\left(X_{\lambda}, p_{\lambda \lambda^{\prime}}, \Lambda\right) \rightarrow \mathbf{Y}=\left(Y_{\mu}, q_{\mu \mu^{\prime}}, M\right)$ is a morphism of inverse systems and let $\mu \in M$. If $\lambda \geq \phi(\mu)$ is a comovability (or uniform co-movability) index of $\mu$ with respect to $\left(f_{\mu}, \phi\right)$, then so is any $\lambda^{\prime}>\lambda$.

Definition 4.5. A morphism of inverse systems $\left(f_{\mu}, \phi\right):\left(\left(X_{\lambda}, *\right), p_{\lambda \lambda^{\prime}}, \Lambda\right)$ $\rightarrow\left(\left(Y_{\mu}, *\right), q_{\mu \mu^{\prime}}, M\right)$ of pointed sets is said to have the Mittag-Leffler property provided every $\mu \in M$ admits a $\lambda \in \Lambda$ with $\lambda \geq \phi(\mu)$ (an ML index for $\mu$ with respect to $\left.\left(f_{\mu}, \phi\right)\right)$ such that for any $\lambda^{\prime} \in \Lambda$ with $\lambda^{\prime} \geq \lambda$ one has

$$
f_{\mu \lambda^{\prime}}\left(X_{\lambda^{\prime}}\right)=f_{\mu \lambda}\left(X_{\lambda}\right) \text {. }
$$

If $\left(f_{\mu}, \phi\right)$ is replaced by $1_{(\mathbf{X}, *)}$ we obtain the Mittag-Leffler property for an inverse system in the category pro-Set $*$ (cf. [12, Ch. II, §6.2]).

THEOREM 4.6. A morphism of inverse systems of pointed sets is comovable if and only if it has the Mittag-Leffler property.

Proof. Let $\left(f_{\mu}, \phi\right):\left(\left(X_{\lambda}, *\right), p_{\lambda \lambda^{\prime}}, \Lambda\right) \rightarrow\left(\left(Y_{\mu}, *\right), q_{\mu \mu^{\prime}}, M\right)$ be a morphism with the Mittag-Leffler property. Then for $\mu \in M$ there is a ML index $\lambda \in \Lambda$ with $\lambda \geq \phi(\mu)$ such that 4.3 holds for each $\lambda^{\prime} \geq \lambda$. We can prove that $\lambda$ is a co-movability index of $\mu$ with respect to $\left(f_{\mu}, \phi\right)$. If $\lambda^{\prime} \geq \phi(\mu)$ and $\lambda^{\prime} \geq \lambda$, then (4.3) defines a map of pointed sets $r:\left(X_{\lambda}, *\right) \rightarrow\left(X_{\lambda^{\prime}}, *\right)$ such that $f_{\mu \lambda^{\prime}} \circ r=f_{\mu \lambda}$. For any other $\lambda^{\prime \prime} \geq \phi(\mu)$, one chooses $\lambda^{\prime \prime \prime} \geq \lambda^{\prime \prime}, \phi(\mu)$ and consider $r^{\prime}: X_{\lambda} \rightarrow X_{\lambda^{\prime \prime \prime}}$ such that $f_{\mu \lambda^{\prime \prime \prime}} \circ r^{\prime}=f_{\mu \lambda}$. Then $r:=p_{\lambda^{\prime \prime} \lambda^{\prime \prime \prime}} \circ r^{\prime}$ satisfies $f_{\mu \lambda^{\prime \prime}} \circ r=f_{\mu \lambda^{\prime \prime}} \circ p_{\lambda^{\prime \prime} \lambda^{\prime \prime \prime}} \circ r^{\prime}=f_{\mu \lambda^{\prime \prime \prime}} \circ r^{\prime}=f_{\mu \lambda}$.

Conversely, assume $\left(f_{\mu}, \phi\right)$ is co-movable. Let $\mu \in M$ and let $\lambda \in \Lambda$ with $\lambda \geq \phi(\mu)$ be a co-movability index of $\mu$ with respect to $\left(f_{\mu}, \phi\right)$. Then for 
$\lambda^{\prime} \geq \lambda$ there exists $r:\left(X_{\lambda}, *\right) \rightarrow\left(X_{\lambda^{\prime}}, *\right)$ such that $f_{\mu \lambda^{\prime}} \circ r=f_{\mu \lambda}$. This implies $f_{\mu \lambda}\left(X_{\lambda}\right) \subseteq f_{\mu \lambda^{\prime}}\left(X_{\lambda^{\prime}}\right)$. The converse inclusion follows from $f_{\mu \lambda} \circ p_{\lambda \lambda^{\prime}}=f_{\mu \lambda^{\prime}}$.

Proposition 4.7. Let $\mathbf{X}=\left(X_{\lambda}, p_{\lambda \lambda^{\prime}}, \Lambda\right)$ and $\mathbf{Y}=\left(Y_{\mu}, q_{\mu \mu^{\prime}}, M\right)$ be inverse systems in a category $\mathcal{C}$, and $\left(f_{\mu}, \phi\right): \mathbf{X} \rightarrow \mathbf{Y}$ a morphism of inverse systems. If $\mathbf{X}$ is a movable [uniformly movable] system and $\mathbf{Y}$ is an arbitrary system, then $\left(f_{\mu}, \phi\right)$ is a co-movable [uniformly co-movable] morphism.

Proof. If $\mu \in M$, then a movability [uniform movability] index $\lambda^{\prime} \in \Lambda$ for $\phi(\mu)$ is a co-movability [uniform co-movability] index for $\mu$ with respect to $\left(f_{\mu}, \phi\right)$.

TheOREM 4.8. An inverse system $\mathbf{X}=\left(X_{\lambda}, p_{\lambda \lambda^{\prime}}, \Lambda\right)$ is movable [uniformly movable] if and only if the identity morphism $1_{\mathbf{X}}$ is co-movable [uniformly co-movable].

Proof. If $\mathbf{X}$ is movable [uniformly movable], then by Proposition 4.7 , the morphism $1_{\mathbf{X}}$ is co-movable [uniformly co-movable]. Conversely, if $1_{\mathbf{X}}$ is co-movable [uniformly co-movable], then a co-movability [uniform co-movability] index of $\lambda \in \Lambda$ with respect to $1_{\mathbf{X}}=\left(1_{X_{\lambda}}, \mathrm{id}_{\Lambda}\right)$ is a movability [uniform movability] index for $\lambda$.

Proposition 4.9. Let $\left(f_{\mu}, \phi\right),\left(f_{\mu}^{\prime}, \phi^{\prime}\right): \mathbf{X}=\left(X_{\lambda}, p_{\lambda \lambda^{\prime}}, \Lambda\right) \rightarrow \mathbf{Y}=$ $\left(Y_{\mu}, q_{\mu \mu^{\prime}}, M\right)$ be equivalent morphisms of inverse systems. If $\left(f_{\mu}, \phi\right)$ is comovable then so is $\left(f_{\mu}^{\prime}, \phi^{\prime}\right)$.

Proof. Let $\mu \in M$ and let $\lambda \in \Lambda$ with $\lambda \geq \phi(\mu)$ be a co-movability index for $\mu$ relative to $\left(f_{\mu}, \phi\right)$. Consider $\bar{\lambda} \in \Lambda$ with $\bar{\lambda} \geq \lambda$ and $\bar{\lambda} \geq \phi(\mu), \phi^{\prime}(\mu)$ such that

$$
f_{\mu \bar{\lambda}}=f_{\mu \bar{\lambda}}^{\prime} .
$$

We now prove that $\bar{\lambda}$ is a co-movability index of $\mu$ relative to $\left(f_{\mu}^{\prime}, \phi^{\prime}\right)$. If $\overline{\lambda^{\prime}} \geq \phi^{\prime}(\mu)$, consider $\lambda^{\prime} \geq \overline{\lambda^{\prime}}, \phi(\mu)$. There exists a morphism $r: X_{\lambda} \rightarrow X_{\lambda^{\prime}}$ such that

$$
f_{\mu \lambda}=f_{\mu \lambda^{\prime}} \circ r
$$

Consider the morphism $\bar{r}: X_{\bar{\lambda}} \rightarrow X_{\overline{\lambda^{\prime}}}$ defined as

$$
\bar{r}=p_{\overline{\lambda^{\prime} \lambda^{\prime}}} \circ r \circ p_{\lambda \bar{\lambda}} \text {. }
$$

Then

$$
\begin{aligned}
f_{\mu \overline{\lambda^{\prime}}}^{\prime} \circ \bar{r} & =f_{\mu}^{\prime} \circ p_{\phi^{\prime}(\mu) \overline{\lambda^{\prime}}} \circ \bar{r}=f_{\mu}^{\prime} \circ p_{\phi^{\prime}(\mu) \overline{\lambda^{\prime}}} \circ p_{\overline{\lambda^{\prime} \lambda^{\prime}}} \circ r \circ p_{\lambda \bar{\lambda}} \\
& =f_{\mu}^{\prime} \circ p_{\phi^{\prime}(\mu) \lambda^{\prime}} \circ r \circ p_{\lambda \bar{\lambda}}=f_{\mu} \circ p_{p h i(\mu) \lambda^{\prime}} \circ r \circ p_{\lambda \bar{\lambda}} \\
& =f_{\mu} \circ p_{\phi(\mu) \lambda} \circ p_{\lambda \bar{\lambda}}=f_{\mu} \circ p_{\phi(\mu) \bar{\lambda}}=f_{\mu}^{\prime} \circ p_{\phi^{\prime}(\mu) \bar{\lambda}},
\end{aligned}
$$

i.e., $f_{\mu \overline{\lambda^{\prime}}}^{\prime} \circ \bar{r}=f_{\mu \bar{\lambda}}^{\prime}$. 
Proposition 4.10. Let $\left(f_{\mu}, \phi\right),\left(f_{\mu}^{\prime}, \phi^{\prime}\right): \mathbf{X}=\left(X_{\lambda}, p_{\lambda \lambda^{\prime}}, \Lambda\right) \rightarrow \mathbf{Y}=$ $\left(Y_{\mu}, q_{\mu \mu^{\prime}}, M\right)$ be equivalent morphisms of inverse systems. If $\left(f_{\mu}, \phi\right)$ is uniformly co-movable then so is $\left(f_{\mu}^{\prime}, \phi^{\prime}\right)$.

Proof. We use the notation from the proof of Proposition 4.9 with the necessary changes: $\lambda$ is a uniform co-movability index for $\mu$ relative to $\left(f_{\mu}, \phi\right)$ and we prove that $\bar{\lambda}$ is a uniform co-movability index of $\mu$ relative to $\left(f_{\mu}^{\prime}, \phi^{\prime}\right)$. The morphism $r$ is replaced by the pro-morphism $\mathbf{r}: X_{\lambda} \rightarrow \mathbf{X}$ satisfying $f_{\mu \lambda}=\mathbf{f}_{\mu} \circ \mathbf{r}$. Then defining $\overline{\mathbf{r}}: X_{\bar{\lambda}} \rightarrow \mathbf{X}$ by $\overline{\mathbf{r}}=\mathbf{r} \circ p_{\lambda \bar{\lambda}}$ it can be verified that $f_{\mu \bar{\lambda}}^{\prime}=\mathbf{f}_{\mu}^{\prime} \circ \overline{\mathbf{r}}$

Thanks to Propositions 4.9 and 4.10 we can introduce the following definition.

Definition 4.11. A morphism $\mathbf{f}: \mathbf{X} \rightarrow \mathbf{Y}$ in a pro-category pro- $\mathcal{C}$ is called co-movable [uniformly co-movable] if $\mathbf{f}$ admits a representation $\left(f_{\mu}, \phi\right)$ : $\mathbf{X} \rightarrow \mathbf{Y}$ which is a co-movable [uniformly co-movable] morphism.

Theorem 4.12. Let $\mathbf{X}=\left(X_{\lambda}, p_{\lambda \lambda^{\prime}}, \Lambda\right), \mathbf{Y}=\left(Y_{\mu}, q_{\mu \mu^{\prime}}, M\right)$ and $\mathbf{Z}=$ $\left(Z_{\nu}, r_{\nu \nu^{\prime}}, N\right)$ be inverse systems in the category $\mathcal{C}$, and let $\mathbf{f}=\left[\left(f_{\mu}, \phi\right)\right]$ : $\mathbf{X} \rightarrow \mathbf{Y}$ and $\mathbf{g}=\left[\left(g_{\nu}, \psi\right)\right]: \mathbf{Y} \rightarrow \mathbf{Z}$ be pro-morphisms. Suppose that $\mathbf{f}$ is a co-movable [uniformly co-movable] pro-morphism. Then the composition $\mathbf{h}=\mathbf{g} \circ \mathbf{f}, \mathbf{h}=\left[\left(h_{\nu}, \chi\right)\right]=\left[\left(g_{\nu} \circ f_{\psi(\nu)}, \phi \circ \psi\right)\right]$, is also a co-movable [uniformly co-movable] pro-morphism.

Proof. Suppose that $\left(f_{\mu}, \phi\right)$ is co-movable. Let $\nu \in N$. Consider $\psi(\nu) \in M$ and let $\lambda \in \Lambda$ with $\lambda \geq \phi(\psi(\nu))$ be a co-movability index of $\psi(\nu)$ relative to $\left(f_{\mu}, \phi\right)$. Then $\lambda$ is a co-movability index of $\nu$ relative to $\left(h_{\nu}, \chi\right)$. Indeed, if $\lambda^{\prime} \geq \chi(\nu)=\phi(\psi(\nu))$ then there is a morphism $r: X_{\lambda} \rightarrow X_{\lambda^{\prime}}$ such that

$$
f_{\psi(\nu) \lambda}=f_{\psi(\nu) \lambda^{\prime}} \circ r
$$

Then

$$
\begin{aligned}
h_{\nu \lambda^{\prime}} \circ r & =h_{\nu} \circ p_{\phi(\psi(\nu)) \lambda^{\prime}} \circ r=g_{\nu} \circ\left(f_{\psi(\nu)} \circ p_{\phi(\psi(\nu)) \lambda^{\prime}}\right) \circ r=g_{\nu} \circ\left(f_{\psi(\nu) \lambda^{\prime}} \circ r\right) \\
& =g_{\nu} \circ f_{\psi(\nu) \lambda}=\left(g_{\nu} \circ f_{\psi(\nu)}\right) \circ p_{\phi(\psi(\nu)) \lambda}=h_{\nu} \circ p_{\chi(\nu) \lambda}=h_{\nu \lambda} .
\end{aligned}
$$

The case of uniform co-movability is similar.

Definition 4.13. A shape morphism $F: X \rightarrow Y$ is co-movable [uniformly co-movable] if it can be represented by a co-movable [uniformly comovable] pro-morphism $\mathbf{f}: \mathbf{X} \rightarrow \mathbf{Y}$.

REMARK 4.14. All properties of co-movability and uniform co-movability of morphisms of inverse systems and of pro-morphisms can be transferred to appropriate properties for shape morphisms and for morphisms in the category $\mathcal{T}$ of a shape theory $\mathbf{S h}_{(\mathcal{T}, \mathcal{P})}$. 
5. Applications. If $\mathbf{p}: X \rightarrow \mathbf{X}, \mathbf{q}: Y \rightarrow \mathbf{Y}$ are inverse limits of $\mathbf{X}, \mathbf{Y} \in$ pro- $\mathcal{C}$, then a morphism $\mathbf{f}: \mathbf{X} \rightarrow \mathbf{Y}$ in pro- $\mathcal{C}$ induces a morphism $f: X \rightarrow Y$ in $\mathcal{C}$ satisfying $\mathbf{q} \circ f=\mathbf{f} \circ \mathbf{p}$. In general, if $f=\lim \mathbf{f}$ is an epimorphism in $\mathcal{C}$, then $\mathbf{f}$ need not be an epimorphism in pro- $\mathcal{C}$ (see [12, Ch. II, §6.1]). But if $\mathbf{Y} \in$ pro- $\mathcal{C}$ is uniformly movable, then the statement is true (see [12, Ch. II, §6.1, Theorem 3] and [14]). The following theorem is a generalization of this result.

Theorem 5.1. Let $\mathbf{f}=\left[\left(f_{\mu}, \phi\right)\right]: \mathbf{X}=\left(X_{\lambda}, p_{\lambda \lambda^{\prime}}, \Lambda\right) \rightarrow \mathbf{Y}=\left(Y_{\mu}, q_{\mu \mu^{\prime}}, M\right)$ be a morphism in pro- $\mathcal{C}$, let $\mathbf{p}=\left[\left(p_{\lambda}\right)\right]: X \rightarrow \mathbf{X}$ and $\mathbf{q}=\left[\left(q_{\mu}\right)\right]: Y \rightarrow \mathbf{Y}$ be inverse limits in $\mathcal{C}$ and let $f=\lim \mathbf{f}: X \rightarrow Y$. If $f$ is an epimorphism in $\mathcal{C}$, $\mathbf{f}$ is uniformly movable and for each $\mu \in M$ the composition $f_{\mu} \circ p_{\phi(\mu)}$ is an epimorphism in $\mathcal{C}$, then $\mathbf{f}$ is an epimorphism in pro-C.

Proof. Let $\mathbf{g}=\left[\left(g_{\nu}, \psi\right)\right], \mathbf{g}^{\prime}=\left[\left(g_{\nu}^{\prime}, \psi^{\prime}\right)\right]: \mathbf{Y} \rightarrow \mathbf{Z}=\left(Z_{\nu}, r_{\nu \nu^{\prime}}, N\right)$ be morphisms in pro-C such that $\mathbf{g} \circ \mathbf{f}=\mathbf{g}^{\prime} \circ \mathbf{f}$. We must prove that $\mathbf{g}=\mathbf{g}^{\prime}$. Since $\mathbf{f} \circ \mathbf{p}=\mathbf{q} \circ f$, it follows that $\mathbf{g} \circ \mathbf{q} \circ f=\mathbf{g}^{\prime} \circ \mathbf{q} \circ f$, i.e., for every $\nu \in N$,

$$
g_{\nu} \circ q_{\psi(\nu)} \circ f=g_{\nu}^{\prime} \circ q_{\psi^{\prime}(\nu)} \circ f,
$$

and therefore

$$
g_{\nu} \circ q_{\psi(\nu)}=g_{\nu}^{\prime} \circ q_{\psi^{\prime}(\nu)} .
$$

Choose $\mu \in M$ with $\mu \geq \psi(\nu), \psi^{\prime}(\nu)$, and let $\lambda \in \Lambda$ with $\lambda \geq \phi(\mu)$ be a uniform movability index of $\mu$ with respect to $\left(f_{\mu}, \phi\right)$. Then there is a morphism $\mathbf{u}: X_{\lambda^{\prime}} \rightarrow \mathbf{Y}$ in pro-C such that

$$
f_{\mu \lambda}=\mathbf{q}_{\mu} \circ \mathbf{u} .
$$

Setting $u=\lim \mathbf{u}: X_{\lambda^{\prime}} \rightarrow Y$, so that $\mathbf{q} \circ u=\mathbf{u}$, we obtain

$$
f_{\mu \lambda}=q_{\mu} \circ u \text {. }
$$

Consequently, $g_{\nu} \circ q_{\psi(\nu) \mu} \circ f_{\mu \lambda}=g_{\nu} \circ q_{\psi(\nu) \mu} \circ f_{\mu \lambda}=g_{\nu} \circ q_{\psi(\nu)} \circ q_{\mu} \circ u=$ $g_{\nu} \circ q_{\psi(\nu)} \circ u$. Similarly,

$$
g_{\nu}^{\prime} \circ q_{\psi^{\prime}(\nu) \mu} \circ f_{\mu} \circ p_{\phi(\mu) \lambda}=g_{\nu}^{\prime} \circ q_{\psi^{\prime}(\nu)} \circ u,
$$

so that (5.1) implies

$$
g_{\nu} \circ q_{\psi(\nu) \mu} \circ f_{\mu \lambda}=g_{\nu}^{\prime} \circ q_{\psi^{\prime}(\nu) \mu} \circ f_{\mu \lambda},
$$

and composing on the right with $p_{\lambda}$ we have

$$
g_{\nu} \circ q_{\psi(\nu) \mu} \circ\left(f_{\mu} \circ p_{\phi(\mu)}\right)=g_{\nu}^{\prime} \circ q_{\psi^{\prime}(\nu) \mu} \circ\left(f_{\mu} \circ p_{\phi(\mu)}\right) .
$$

By the hypothesis this implies

$$
g_{\nu} \circ q_{\psi(\nu) \mu}=g_{\nu}^{\prime} \circ q_{\psi^{\prime}(\nu) \mu},
$$

which shows that $\left(g_{\nu}, \psi\right) \sim\left(g_{\nu}^{\prime}, \psi^{\prime}\right)$ and therefore $\mathbf{g}=\mathbf{g}^{\prime}$ in pro-C . 
REMARK 5.2. The additional condition from Theorem 5.1, that $f_{\mu} \circ p_{\phi(\mu)}$ is an epimorphism, depends only on the pro-morphism $\mathbf{f}$. Indeed, if $\left(f_{\mu}^{\prime}, \phi^{\prime}\right) \sim$ $\left(f_{\mu}, \phi\right)$, then for every $\mu \in M$ there exists $\lambda \geq \phi(\mu), \phi^{\prime}(\mu)$ such that we have $f_{\mu} \circ p_{\phi(\mu) \lambda}=f_{\mu}^{\prime} \circ p_{\phi^{\prime}(\mu) \lambda}$. From this, by composing on the right with $p_{\lambda}$, we obtain $f_{\mu} \circ p_{\phi(\mu)}=f_{\mu}^{\prime} \circ p_{\phi^{\prime}(\mu)}$.

We recall that if $\mathcal{C}$ is a category with zero objects, then a morphism $f: A \rightarrow B$ of $C$ is a weak epimorphism if $u \circ f=0$ implies $u=0$. Weakened versions of the categorical notions of monomorphism and epimorphism have proved to be of some interest in pointed homotopy theory. In 1986, J. Roitberg [18] used the properties of a remarkable group discovered by G. Higman [10] to exhibit examples in the pointed homotopy category of weak epimorphisms which are not epimorphisms. Obviously, pro-Grp and pro-HTop* are categories with zero objects. Generally, if a category $\mathcal{C}$ is with zero objects then the pro-category pro- $\mathcal{C}$ is with zero objects. Now the proof of Theorem 5.1 is perfectly adaptable to yield the following weakened version.

THEOREM 5.3. With the notation of Theorem 5.1 and supposing that the category $\mathcal{C}$ is with zero objects, $f$ is a weak epimorphism in $\mathcal{C}$ and $\mathbf{f}$ is uniformly movable such that for each $\mu \in M$ the composition $f_{\mu} \circ p_{\phi(\mu)}$ is a weak epimorphism in $\mathcal{C}$, then $\mathbf{f}$ is a weak epimorphism in pro-C.

REMARK 5.4. If $\mathbf{Y}$ is movable, then we obtain a weakened version of Moszyńska's Theorem [14] (see also [12, Ch. II, §6.1, Theorem 3]).

Corollary 5.5. Let $\mathbf{f}=\left[\left(f_{\mu}\right)\right]: X \rightarrow \mathbf{Y}=\left(Y_{\mu}, q_{\mu \mu^{\prime}}, M\right)$ be a morphism in pro-C and $f=\lim \mathbf{f}: X \rightarrow Y=\lim \mathbf{Y}$. If $f$ and every $f_{\mu}: X \rightarrow Y_{\mu}$, $\mu \in M$, are epimorphisms [weak epimorphisms] in $\mathcal{C}$, then $\mathbf{f}$ is an epimorphism [a weak epimorphism $]$ in pro-C.

In particular, if $\mathbf{p}=\left(p_{\lambda}\right): X \rightarrow \mathbf{X}=\left(X_{\lambda}, p_{\lambda \lambda^{\prime}}, \Lambda\right)$ is an inverse limit with all projections $p_{\lambda}$ being epimorphisms [weak epimorphisms] in $\mathcal{C}$, then $\mathbf{p}$ is an epimorphism [weak epimorphism] in pro-C.

Now we can state two variants of Dydak's infinite-dimensional Whitehead theorem in shape theory [5].

TheOREM 5.6. Let $F:(X, *) \rightarrow(Y, *)$ be a weak shape equivalence of pointed connected topological spaces. Suppose that $X$ is of finite shape dimension, and $F$ is a movable pointed shape domination. Then $F$ is a pointed shape equivalence.

Proof. $F$ being a pointed shape domination means that there exists a shape morphism $G:(Y, *) \rightarrow(X, *)$ such that $F G=1_{(Y, *)}$. Then we can apply Theorem 2.11 to deduce that $(Y, *)$ is movable. Now the conclusion follows from [12, Ch. II, §7.2, Theorem 6(i)]. 
Theorem 5.7. Let $F:(X, *) \rightarrow(Y, *)$ be a weak shape equivalence of pointed connected topological spaces. Suppose that $Y$ is of finite shape dimension, and $F$ is a co-movable pointed shape morphism which has a left inverse. Then $F$ is a pointed shape equivalence.

Proof. By Theorem 4.12 in the shape version, $(X, *)$ is movable. Then the conclusion follows from [12, Ch. II, §7.2, Theorem 6(ii)].

Acknowledgements. The authors are grateful to the referee for valuable suggestions.

\section{References}

[1] T. A. Avakyan and P. S. Gevorgyan, Strong movable categories and strong movability of topological spaces, J. Contemp. Math. Anal. Armen. Acad. Sci. 45 (2010), 52-59.

[2] K. Borsuk, On movable compacta, Fund. Math. 66 (1969/70), 137-146.

[3] Z. Čerin, Lefschetz movable maps, J. Math. Pures Appl. 72 (1993), 81-103.

[4] J.-M. Cordier and T. Porter, Shape Theory: Categorical Methods of Approximation, Ellis Horwood, 1989.

[5] J. Dydak, The Whitehead and Smale theorems in shape theory, Dissertationes Math. 156 (1979), 55 pp.

[6] A. D. Edwards and P. Tulley McAuley, The shape of a map, Fund. Math. 96 (1977), $195-210$.

[7] P. S. Gevorgyan, Movable categories, Glas. Mat. Ser. III 38 (2003), 177-183.

[8] P. S. Gevorgyan, On a movability criterion, Math. Notes 71 (2002), 281-284.

[9] P. S. Gevorgyan and I. Pop, Uniformly movable categories and uniform movability of topological spaces, Bull. Polish Acad. Sci. Math. 55 (2007), 229-242.

[10] G. Higman, A finitely related group with an isomorphic proper factor group, J. London Math. Soc. 26 (1951), 59-61.

[11] S. Mardešić and J. Segal, Movable compacta and ANR-systems, Bull. Acad. Polon. Sci. Sér. Sci. Math. Astronom. Phys. 18 (1970), 649-654.

[12] S. Mardešić and J. Segal, Shape Theory. The Inverse System Approach, NorthHolland, 1982.

[13] K. Morita, On shapes of topological spaces, Fund. Math. 86 (1975), 251-259.

[14] M. Moszyńska, Uniformly movable compact spaces and their algebraic properties, Fund. Math. 77 (1972), 125-144.

[15] I. Pop, A categorical notion of movability, An. Ştiinţ. Univ. "Al. I. Cuza" Iaşi Mat. (N.S.) 49 (2003), 327-341.

[16] I. Pop, On movability of pro-morphisms, An. Univ. Vest Timişoara Ser. Mat.-Inform. 48 (2010), 223-238.

[17] I. Pop, New categorical approaches to movability in shape theory, An. Ştiinţ. Univ. "Al. I. Cuza" Iaşi Mat. 59 (2013), 25-42.

[18] J. Roitberg, On weak epimorphisms in homotopy theory, Pacific J. Math. 121 (1986), $183-187$. 
[19] T. Yagasaki, Fiber shape theory, Tsukuba J. Math. 9 (1985), 261-277.

[20] T. Yagasaki, Movability of maps and shape fibrations, Glas. Mat. 21 (1986), 153-177.

P. S. Gevorgyan

Faculty of Mathematics

Moscow State Pedagogical University

88, Vernadskogo St.

Moscow, Russia

E-mail: pgev@yandex.ru
I. Pop

Faculty of Mathematics

"Al. I. Cuza" University 700505 Iaşi, Romania

E-mail: ioanpop@uaic.ro 
